\title{
Evaluation of Total White Blood Cells in Obese Subject in Khartoum State 2017
}

\section{Wala M Mostafa ${ }^{1}$, Ibrahim A Ali ${ }^{1 *}$, Omer A Musa ${ }^{1}$ and Elmutaz H Taha $^{2}$}

${ }^{1}$ Department of Physiology, The National Ribat University, Sudan

${ }^{2}$ Department of Physiology, University of Dongola, Sudan

*Corresponding author: Ibrahim A Ali, Department of Physiology, Faculty of medicine, The National Ribat University, Khartoum Box 55, Sudan, Tel: 24912377276; Email: Hemamedicine@gmail.com

\section{Research Article}

Volume 3 Issue 2

Received Date: October 09, 2019

Published Date: October 21, 2019

DOI: $10.23880 /$ hij-16000148

\section{Abstract}

Background: This research aimed to investigate the effect of obesity WBCs count and to compare WBCs count of obese subjects early in the morning and in the late evening to explore the effect of normal activates on TWBCs.

Methods: Across sectional facility-based study was conducted in the Khartoum state during the period of Sep 2017 to Nov 2017 among Sudanese obese subjects of age between 15-45 years old

Result: There is variation in number of TWBS early in the morning comparing to late in the evening.The mean value of TWBCs for morning was 6.29 with standard deviation of 1.75 and the mean of TWBS result for evening is 7.37 with standard deviation of 1.78 . The pair sample $T$ test was significant with a $P$ value 0.00

Conclusion: There is significant increase in number of TWBCs at the end of the day in obese subject, the cause may be due to the normal activity of obese considered as exercise.

Keywords: BMI; White blood cells; World health organization

Abbreviations: CRP: C-reactive Protein; WHO: World Health Organization; WBCs: White Blood Cells; IGT: Impaired Glucose Tolerance; BMI: Body Mass Index.

\section{Introduction}

In the last decade, obesity has become one of the most significant public health crises in the United States for both adults and children [1]. According to world health, organization (WHO), obesity is classified as chronic and severe disease in developed and under developing countries, affecting both adult and children. Obesity is associated with increased incidence of type 2 diabetes mellitus, hypertension, coronary heart disease, arthritis, sleep apnea, and certain form of cancer. Recent research data suggest that the global incidence of obesity has increased more than $75 \%$ since 1980 [2]. The normal physiological range for white blood cells and differential count are essential for diagnosis, treatment, follow up and screening. The WBCs count of Sudanese people was found to be lower than that of Caucasians and similar to reports from other African countries; Taha, et al. performed a study and they found the mean of $\mathrm{WBC}$ count was $5.1 \pm 1.5 \times 103 / \mu \mathrm{l}$ with a range of $3.6 \times 103 / \mu \mathrm{l}$ to 6.6 
$\times 103 / \mu$ l. Also, they reported in their study the WBCs count was directly proportional to BMI [3].

WBCs count in obese subjects were significantly higher than in non-obese subjects [4]. The positive relationship between WBC count, insulin resistance, hypertension, and cardiovascular disease has been observed in several studies [5]. Adipose tissue is a major source of inflammatory factors, such as IL-6 and Creactive protein (CRP), which also are well established markers of systemic inflammation [6]. Pro-inflammatory cytokines, such as interleukin 6 (IL-6) and interleukin 8 (IL-8), are important inducers of WBCs production [7]. Higher WBC counts may be associated with the development of retinopathy, albuminuria, and peripheral arterial disease [8].

White blood cells (WBCs) count is elevated in obesity and is risk factor for atherosclerosis and is present in impaired glucose tolerance (IGT) which is associated with Marco and Micro-angiopathic complication in type 2 diabetes, the effect is more marked in obese patient [9]. Research has shown that obese adults, (BMI-- $30 \mathrm{~kg} / \mathrm{m}^{2}$ ), have elevated total leukocytes [3] elevation of leukocytes appears to be related to monocytes [10]; however, neutrophils, eosinophils, and lymphocytes may be elevated [11]. And weight loss is important in reducing WBCs count in morbid obesity, simple or complicated by IGT or type 2 Diabetes [9]. Total leukocyte and monocyte count were significantly increased in obese young adult, total leucocyte count was associated with liver enzyme level, insulin resistance as well as visceral and subcutaneous fat thickness.

Neutrophil count was associated with insulin resistance; lymphocyte count was associated with serum liver enzyme, insulin resistance, and dyslipidemia. Monocyte count was associated with serum liver enzyme insulin resistance, visceral and subcutaneous fat thickness, body fat mass, and percentage body fat. Chronic low-grade systemic inflammation is associated with obesity related complication such as non-alcoholic fatty liver disease, insulin resistance and dyslipidemia in young adult [12]. Increased total WBCs and WBCs differential count particularly lymphocyte and granulocyte are associated with risk of transition of type 2 diabetes in obese Mexican American, after adjusting for other potential confounders [10]. A high interpersonal variation in white blood cell (WBC) counts has also been reported in physically active individuals [13] physical exercise may promote changes in the immune system [14] and can alter the number and function of leukocytes [15]. The degree of WBC trafficking depends on the intensity and duration of exercise [16]. It has been postulated that exercise increases stress-induced changes in the immuneneuroendocrine axis and in the circulating levels of metabolites that directly influence the function of immune cells [17]. This research aimed to investigate the effect of obesity WBCs count and compare WBCs count of obese subjects early in the morning and in the late evening to explore the effect of normal activates on TWBCs.

\section{Methods}

A cross sectional facility-based study was conducted in the Khartoum - Sudan during Sep 2017 to Nov 2017 among the Normal Sudanese obese subject. Medical record of 20 adult subject were reviewed all of them considered obese subject. Information obtained from the medical record include age gender height, weight and body mass index (BMI). BMI was calculated by dividing (weight in $\mathrm{kg}$ ) over (height in $\mathrm{m}^{2}$ ). Subject with BMI above 30 were considered obese while those with BMI less 30 were non-obese according to WHO. Twenty subjects were included; five of them were males and fifteen were females with age between (15-45) years old. Arterial blood pressure was checked for each volunteer to exclude hypertension. Blood samples were analyzed for TWBCs by SPSS version 24 for statistical analysis.

\section{Results}

Within the framework of study of obese subject, we count TWBCs in early of morning and late at evening among 20 obese subject who BMI 30 or more than 30 . The mean of age was 45 with standard deviation of 8.36 years (Table 1). The mean of body mass index of participants was $30.4 \pm 5.0$ with minimums of 30.4 and maximums of 46.6. The mean value of TWBCs for morning was 6.29 with standard deviation of 1.75 and the mean of TWBS result for evening is 7.37 with standard deviation of 1.78 (Table 2). The pair sample T test was significant with a $\mathrm{P}$ value 0.00 (Table 3 ).

\begin{tabular}{|c|c|c|c|c|c|}
\hline Variable & N & Minimum & Maximum & Mean & Std. Deviation \\
\hline BMI & 20 & 30.4 & 46.6 & 35.6515 & 5.00823 \\
\hline Age & 20 & 15 & 45 & 31.7 & 8.367 \\
\hline
\end{tabular}

Table 1: Descriptive Statistics of age and BMI. 


\begin{tabular}{|c|c|c|c|c|c|}
\hline Variable & N & Minimum & Maximum & Mean & Std. Deviation \\
\hline BMI & 20 & 30.4 & 46.6 & 35.6515 & 5.00823 \\
\hline Age & 20 & 15 & 45 & 31.7 & 8.367 \\
\hline
\end{tabular}

Table 2: TWBC reference intervals in early morning and late evening among obese subject.

\begin{tabular}{|c|c|c|c|}
\hline Sample & Difference (\%) & T & P. value \\
\hline TWBCs morning & $23 \% \pm 5.7 \%$ & 4.62 & 0 \\
\hline TWBCs Evening & & 0 \\
\hline
\end{tabular}

Table 3: Paired Samples Statistics.

\section{Discussion}

In this study, we count the number of TWBCs in obese subject and compare it early in the morning and late in the evening, and to observe the relation between BMI and TWBCs in cross sectional study. Our study was showed that the mean TWBCs at morning is significantly different than in evening and there is significant increase in TWBCs at evening. More recent study linked the TWBCs to obesity, in recent ongoing study on normal hematological study in Sudanese population it had been observed that the WBCs was found to be more in obese subjects, as exercise is known to increase the WBC count physiologically it has been suggested that the normal daily movement for obese subject could be take an increase for them [3].

\section{Conclusion}

There is significant increase in number of TWBCs at the end of the day in obese subject, the cause may be due to the normal activity of obese may considered as exercise. TWBCs is affected by normal activates in obese subject. The current study comprised a single study thus additional design study is required. The result providing supporting evidence for recent report that recommended measured TWBCs level at early in the morning and late of the day in obese subject who BMI $\geq 30$. Also, Additional study is required to measured TWBCs level at early in the morning and late of the day in pregnant ladies.

\section{References}

1. Mackay AP, Lingerbut LA, Duran CR (2000) Adolescent Health Chartbook. National Center for Health Statistics: Hyansville MD.

2. Nieto FJ, Szoklo M, Folsom AR, Rock R, Mercuri M (1992) Leukocyte count correlates in middle-aged adults: the Atherosclerosis Risk in Communities (ARIC) Study. Am J Epidemiol 136(5): 525-537.
3. Taha EH, Elshiekh M, Alborai A, Hajo EY, Hussein A, et al. (2018) Normal range of white blood cells and differential count of Sudanese in Khartoum state. Int J Adv Med 5(4): 784-787.

4. Farhangi MA, Keshavarz SA, Eshraghian M, Ostadrahimi A, Saboor-Yaraghi AA (2013) White Blood Cell Count in Women: Relation to Inflammatory Biomarkers, haematological Profiles, Visceral Adiposity, and Other Cardiovascular Risk Factors. J Health Popul Nutr 31(1): 58-64.

5. Karthikeyan VJ, Lip GY (2006) White blood cell count and hypertension. J Hum Hypertens 20: 310-312.

6. Fantuzzi G (2005) Adipose tissue, adipokines, and inflammation. J Allergy Clin Immunol 115(5): 911919.

7. Ohshita K, Yamane K, Hanafusa M, Mori H, Mito K, et al. (2004) Elevated white blood cell count in subjects with impaired glucose tolerance. Diabetes Care 27(2): 491-496.

8. Moradi S, Kerman SR, Rohani F, Salari F (2012) Association between diabetes complications and leukocyte counts in Iranian patients. J Inflamm Res 5: 7-11.

9. Anna Veronelli, Marco Laner, Roberto Ranieri, Diana Koprivec, Debora Vardaro, et al. (2004) White blood cells in obesity and diabetes. Diabetes care 27(10): 2501-2502.

10. Kullo IJ, Hensrud DD, Allison TG (2002) Comparison of numbers of circulating blood monocytes in men grouped by body mass index $(<25,25$ to $<30$, $>$ or =30). Am J Cardiol 89(12): 1441-1443.

11. Nieman DC, Henson DA, Nehlsen Cannarella SL, Ekkens M, Utter AC, et al. (1999) Influence of obesity on immune function. J Am Diet Assoc 99(3): 294-299. 
12. Schwartz J, Weiss ST (1991) Host and environmental factors influencing the peripheral blood leukocyte count. Am J Epidemiol 134(12): 1402-1409.

13. Nunes LA, Brenzikofer R, de Macedo DV (2010) Reference change values of blood analytes from physically active subjects. Eur J Appl Physiol 110(1): 191-198.

14. Navalta JW, Sedlock DA, Park KS (2007) Effect of exercise intensity on exercise-induced lymphocyte apoptosis. Int J Sports Med 28(6): 539-542.

15. Green KJ, Rowbottom DG, Mackinnon LT (2002) Exercise and T-lymphocyte function: a comparison of proliferation in PBMC and NK cell-depleted PBMC culture. J Appl Physiol 92(6): 2390-2395.

16. Edwards KM, Burns VE, Carroll D, Drayson M, Ring C (2007) The acute stress-induced immunoenhancement hypothesis. Exerc Sport Sci Rev 35(3): 150-155.

17. Risoy BA, Raastad T, Hallen J, Lappegard KT, Baeverfjord K, et al. (2003) Delayed leukocytosis after hard strength and endurance exercise: aspects of regulatory mechanisms. BMC Physiol 3: 14. 